\title{
Hereditary Spastic Paraplegia
}

National Institute of Neurological Disorders and Stroke (NINDS)

\section{Source}

National Institute of Neurological Disorders and Stroke (NINDS). Hereditary Spastic Paraplegia Information Page.

Hereditary spastic paraplegia (HSP), also called familial spastic paraparesis (FSP), refers to a group of inherited disorders that are characterized by progressive weakness and spasticity (stiffness) of the legs. Early in the disease course, there may be mild gait difficulties and stiffness. These symptoms typically slowly progress so that eventually individuals with HSP may require the assistance of a cane, walker, or wheelchair. Though the primary features of "pure" HSP are prog ressive lower limb spasticity and weakness, complicated forms may be accompanied by other symptoms. These additional symptoms include impaired vision due to cataracts and problems with the optic nerve and retina of the eye, ataxia (lack of muscle coordination), epilepsy, cog nitive impairment, peripheral neuropathy, and deafness. The diagnosis of HSP is primarily by neurological examination and testing to rule out other disorders. Brain MRI abnormalities, such as a thin corpus callosum, may be seen in some of the complicated forms of HSP. Several genetic mutations have been identified which underlie various forms of HSP, and specialized genetic testing and diagnosis are available at some medical centers. HSP has several forms of inheritance. Not all children in a family will necessarily develop symptoms, although they may be carriers of the abnormal gene. Symptoms may beg in in childhood or adulthood, depending on the particular HSP gene involved. 